\title{
СИММЕТРИЯ, АСИММЕТРИЯ, ДИССИММЕТРИЯ, АНТИСИММЕТРИЯ
}

Войтеховский Ю.Л., Степенщиков Д.Г.

Геологический институт КНЦРАН, Anamumbl,woyt@geoksc.apatity.ru

При известной сложности процедуры и заведомой критикуемости результата начать следует с определений. Симметрия - самосовмещающее движение фигуры (тела, среды) при сохранении метрики (расстояний между соответствующими точками). Асимметрия - отсутствие нетривиальных элементов симметрии. Диссимметрия (по П. Кюри, а не Л. Пастеру) - выпадение некоторых элементов симметрии тела из его характеристической (внутренне присущей, максимально возможной, проявляющейся в идеальных условиях) группы симметрии под действием среды. Предел диссимметрии - асимметрия. Антисимметрия - энантиоморфизм (диссимметрия по Л. Пастеру) плюс перекрашивание, то есть изменение физического свойства фигуры (тела) на противоположное (цвет: чёрный $v s$. белый, заряд: + vs. -). Несмотря на богатую историю исследований, в этой междисциплинарной области (особенно на стыках разделов), есть нерешённые вопросы и точки роста.

\section{Симлетрия и асимметрия}

Теория симметрии [6] - один из краеугольных камней в наших представлениях о строении и стабильности геологических систем. В основном это точечные группы симметрии (т.г.с.) и фёдоровские группы при описании минеральных индивидов, а также предельные т.г.с. П. Кюри [3] при описании сред: $\infty$ (вращающийся конус) - среда с полярным направлением, $\infty \mathrm{m}$ (неподвижный конус) - однородное электрическое поле, $\infty / \mathrm{m}$ (вращающийся цилиндр) - однородное магнитное поле, $\infty 2$ (скрученный цилиндр) - оптически активная анизотропная среда, $\infty / \mathrm{mm}$ (неподвижный цилиндр) - однородное поле одноосных механических напряжений, $/ \infty$ (вращающая в каждой точке сфера) - оптически активная изотропная среда, $\infty / \infty \mathrm{m}$ (покоящаяся сфера) - однородное скалярное поле.

Ряд всероссийских (с международным участием) конференций, проведенных в Институте геологии Коми НЦ УрО РАН под титулом «Биоминеральные взаимодействия», показал, что геологи приняли представления В.И. Вернадского [1] о живом веществе как планетарном явлении. Но живое вещество как среда не характеризуется ни одной из перечисленных выше предельных т.г.с. Его суть - непрерывное изменение в каждой точке, согласованное (иногда не согласованное) в неоднородной системе живого организма более сложно, чем это указано для физических полей. Живое вещество как среда - неоднородное скалярное потенциальное поле. Его следует характеризовать предельной т.г.с., более асимметричной, чем таковая для среды с полярным направлением (ळ). По-видимому, это вполне подтверждается крайней искажённостью биоминеральных индивидов и агрегатов в соответствии с принципом диссимметрии П. Кюри [3].

Можно ли определить асимметрию как отсутствие нетривиальной симметрии без отрицающего «а», через эквивалентное свойство фигуры? По крайней мере для выпуклых полиэдров в комбинаторном приближении (с точностью до непрерывной деформации, без сохранения метрики) это возможно. В статье [9] для этого использованы рёберные графы и их матрицы смежности. Покажем идею метода на примере тетраэдра (рис. 1). Из-за малого числа вершин и высокой симметрии при любой нумерации его вершин получим ту же матрицу смежности. Из-за её симметрии для фиксации графа достаточно верхнего треугольника. Выписав его построчно, получим двоичный (в других случаях появятся нули) код (имя) тетраэдра. Ради краткости переведём его в десятичную систему: $111111 \rightarrow 63$. Обратным рассуждением по имени рёберный граф любого выпуклого полиэдра строится однозначно. Но тогда в имени должны содержаться все его топологические (комбинаторные) свойства.

С ростом числа вершин n число их различных нумераций растёт как $\mathrm{n}$ ! Неужели указанный алгоритм всегда даёт одно имя выпуклого полиэдра? Отнюдь, число его различных имён равно 

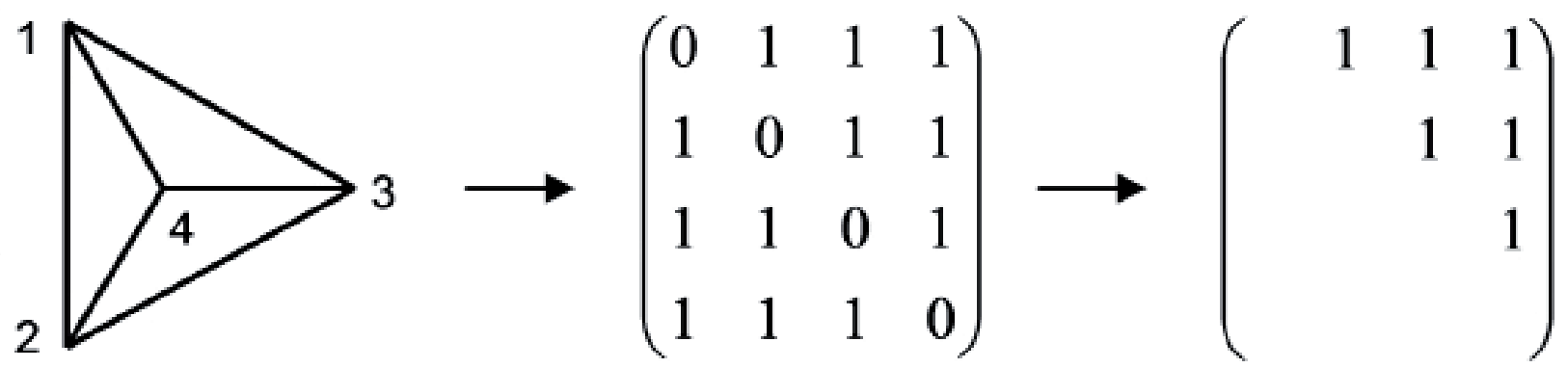

Рис. 1. Рёберный граф и матрица смежности тетраэдра.

n. Автором доказан и опубликован ряд теорем: о связи указанного алгоритма именования выпуклых полиэдров с алгоритмом Е.С. Фёдорова генерирования их комбинаторного многообразия из тетраэдра; о типах n-вершинников с минимальным и максимальным именами; о непересечении диапазонов имён n-вершинников для последовательных n; о характере разбегания диапазонов имён на числовой прямой с ростом $n$. Тем самым переброшен мост от классической кристалломорфологии к комбинаторной теории выпуклых полиэдров, что должно способствовать обогащению обеих дисциплин.

\section{Диссимметрия и антисимметрия}

Диссимметрия - традиционное кристаллографо-минералогическое направление исследований учёных Санкт-Петербургского горного института. Автор неоднократно обращался к нему под влиянием работ И.И. Шафрановского. Антисимметрия представляет собой нечто иное, из диссимметрии не выводимое, обычно определяемое как энантиоморфизм плюс перекрашивание, то есть изменение физического свойства тела на противоположное $[5,7]$. При этом физическое свойство возникает в рассуждении совершенно неожиданно, инородно. Автором найден более органичный смысловой переход от диссимметрии к антисимметрии, не требующий вовлечения физического свойства в сугубо геометрическое рассмотрение.

В статье [2] определена реальная кристаллографическая простая форма (р.к.п.ф.) как выпуклый полиэдр, ограниченный некоторыми гранями исходной простой формы, находящимися в правильной ориентации на любом расстоянии от начала координат. Некоторые грани можно удалять «в бесконечность». Компьютерное перечисление р.к.п.ф. для любых к.п.ф. и их комбинаций даёт инструмент для описания диссимметричных кристаллов, сформировавшихся даже в сильно анизотропных полях. Октаэдр порождает 30 (включая его) комбинаторно различных р.к.п.ф. $(\mathbf{1}, \boldsymbol{m}, \boldsymbol{m m} \mathbf{2}$, $2 / m, 3 m, m m m, \overline{3} m, \overline{4} 3 m)$ [8], ромбододекаэдр - $625(1, m, 2, \overline{1}, m m 2,222,2 / m, 32, \overline{4} 2 m, m m m$, $\overline{3} \boldsymbol{m}, 4 / \boldsymbol{m m m}$ ) [10]. В обоих случаях все р.к.п.ф. относятся к подгруппам предельно высокой т.г.с. $(\boldsymbol{m} \overline{\mathbf{3}} \boldsymbol{m})$ исходных форм. В общем случае это не обязательно. Но заметим, что вопрос о соотношениях т.г.с. исходного кристаллического полиэдра и его р.к.п.ф. не изучен.
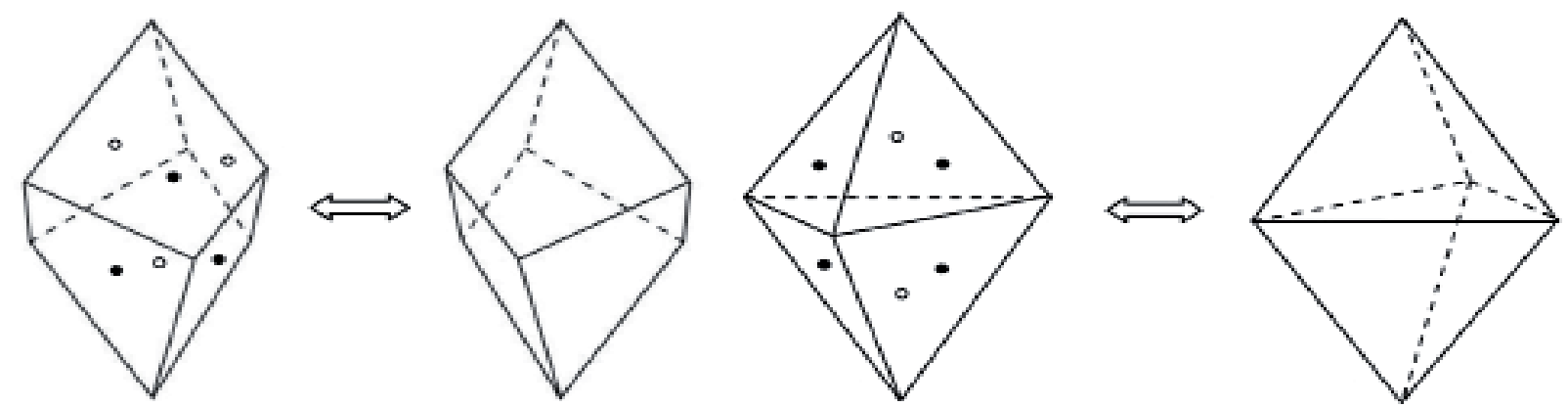

Рис. 2. Антисимметричный тригональный трапецоэдр (слева) и перекрашенная тригональная дипирамида (справа). Внутренние нормали указаны чёрными (на передних гранях) и белыми (на задних гранях) кружками, внешние векторы-нормали не указаны. 

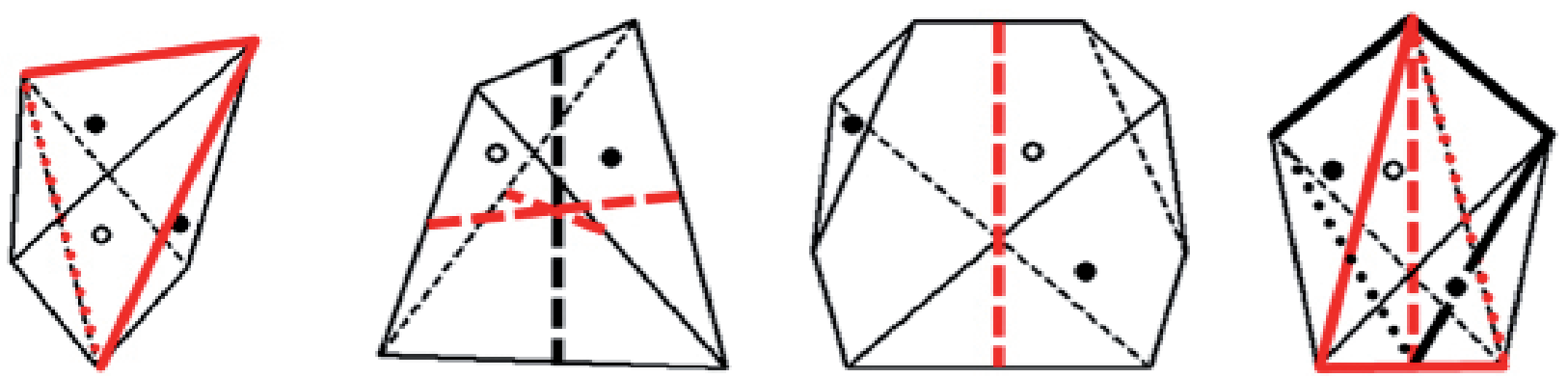

Рис. 3. Антисимметричные полиэдры, порождаемые движениями граней тригонального трапецоэдра (слева, т.г.с. $\boldsymbol{m}$ ') и тригональной дипирамиды (справа, $\left.\mathbf{2}^{\prime} \mathbf{2}^{\prime} \mathbf{2}, \mathbf{2}^{\prime}, \boldsymbol{m} \boldsymbol{m} \mathbf{2} \mathbf{2}\right)$. Плоскости и оси антисимметрии показаны красным цветом.

При генерировании р.к.п.ф. движение граней вдоль нормалей допускалось лишь вовне и моделировало анизотропный рост кристалла. Но что будет, если переместить грани на другую сторону от начала координат на то же расстояние? Если исходный полиэдр примитивен или аксиален (то есть имеет лишь поворотные оси), то полученный (инвертированный) будет ему энантиоморфен. С учётом изменения ориентировок внешних векторов-нормалей на противоположные получим антисимметричный (вывернутый наизнанку, по А.В. Шубникову) полиэдр. Если исходный полиэдр содержит элементы симметрии 2-го рода, то инвертированный совместимо равен ему и лишь «перекрашен», то есть имеет противоположные ориентировки векторов-нормалей к граням (рис. 2).

Усложним задачу, переместив на другую сторону от начала координат любое число граней на любое расстояние. Для примера возьмём тригональные трапецоэдр (32) и дипирамиду ( $\overline{\boldsymbol{6}} \boldsymbol{m} 2)$. Первый имеет лишь элементы симметрии 1-го рода, второй - и 2-го рода (но не имеет параллельных граней, что важно). Указанными выше движениями граней тригонального трапецоэдра получим 63 комбинаторно различных полиэдра, из которых 1 имеет шубниковскую т.г.с. $\boldsymbol{m}$ ', остальные - классические 1 и 2 . Из тригональной дипирамиды получим 36 комбинаторно различных полиэдров, из которых 3 имеют шубниковские т.г.с. 2'2'2, 2' и $\boldsymbol{m} \boldsymbol{m}$ '2', остальные - классические 1, 2 и $\boldsymbol{m}$ (рис. 3).

Обращает внимание то, что $\boldsymbol{m}$ - не подгруппа т.г.с. 32 тригонального трапецоэдра, 222 - не подгруппа т.г.с. $\overline{\boldsymbol{6}} \boldsymbol{m} \mathbf{2}$ тригональной дипирамиды. То есть, в геометрическом отношении т.г.с. полученных полиэдров не являются подгруппами т.г.с. исходных. Это находит простое объяснение. Часть граней антисимметричных полиэдров взята из граней исходного, часть - из граней инвертированного полиэдра. По сути, антисимметричные полиэдры составлены из граней комбинированного (исходный плюс инвертированный) полиэдра с удвоенным числом граней (здесь важно отсутствие параллельных граней у исходного полиэдра), заведомо обладающего центрально-симметричной
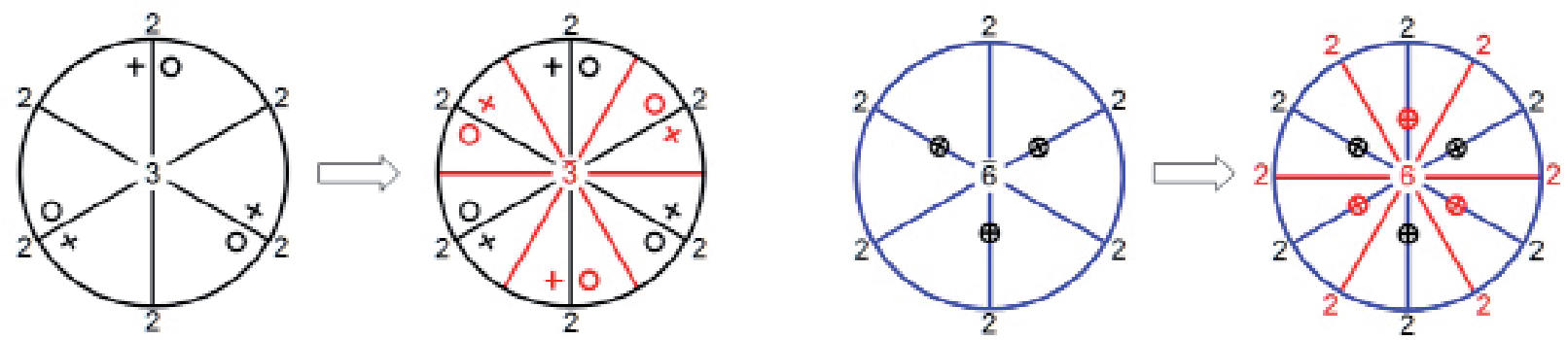

Рис. 4. Исходные, инвертированные и комбинированные выпуклые полиэдры на стереографических проекциях. Тригональный трапецоэдр (32) порождает дитригональный скаленоэдр ( $\overline{\mathbf{3}^{\prime} \boldsymbol{m}} \boldsymbol{\prime}$, слева), тригональная дипирамида ( $\overline{\boldsymbol{6}} \boldsymbol{m} \mathbf{2})$ - гексагональную дипирамиду ( $\boldsymbol{6}^{\prime} / \mathbf{m m} \boldsymbol{m}$ ', справа). Грани даны чёрными (исходные) и красными (инвертированные) крестиками (передняя полусфера) и кружками (задняя полусфера), оси симметрии $(2,3, \overline{6})$ и антисимметрии $(2, \overline{3}, \boldsymbol{\sigma})$ - чёрными и красными цифрами, плоскости симметрии и антисимметрии - синими и красными линиями, соответственно. 
шубниковской т.г.с. Исходный и инвертированный полиэдры гемиэдричны по отношению к комбинированному. (Приведенное описание сходно с описанием двойника [4, с. 188-189], отвечающего нашему комбинированному полиэдру. Разница в том, что нас интересует не он, а получаемые из него антисимметричные полиэдры с числом граней не более чем у исходного.) Теперь очевидно, что $\boldsymbol{m}$ ' - подгруппа шубниковской т.г.с. дитригонального скаленоэдра $\left(\overline{\mathbf{3}^{\prime}} \boldsymbol{\boldsymbol { m }} \boldsymbol{\prime}\right)$, порождаемого тригональным трапецоэдром, а 2'2'2, 2' и $\mathbf{m} \boldsymbol{m}$ '2' - подгруппы шубниковской т.г.с. гексагональной дипирамиды ( $\left.\boldsymbol{\sigma}^{\prime} \mathbf{m} \mathbf{m} \boldsymbol{m} \mathbf{\prime}\right)$, порождаемой тригональной дипирамидой (рис. 4).

\section{Выводы}

В развитие темы «Биоминеральные взаимодействия» предложено характеризовать живое вещество как среду минералообразования (неоднородное скалярное потенциальное поле) с предельной т.г.с., более асимметричной, чем таковая среды с полярным направлением.

Предложен алгоритм численного кодирования (именования) комбинаторного типа выпуклого полиэдра через матрицу смежности его рёберного графа. Анализ матрицы смежности позволяет вычислить п.г.а. и даже т.г.с. полиэдра.

Антисимметричные формы получены в результате радикальной (с переводом части граней вдоль нормалей по другую сторону от начала координат) диссимметризации выпуклых полиэдров, как содержащих, так и не содержащих элементы симметрии 2-го рода.

Работа выполнена в рамках темы НИР ГИ КНЦ РАН № 0231-2015-0001.

\section{Литература}

1. Вернадский В.И. Химическое строение биосферы Земли и её окружения. М.: Наука, 1987. 340 c.

2. Войтеховский Ю.Л., Степенщиков Д.Г. Реальные кристаллографические простые формы // Зап. ВМО. 2004. № 2. С. 112-120.

3. Кюри П. О симметрии в физических явлениях: симметрия электрического и магнитного полей // Избр. труды. М.: Наука, 1966. С. 95-113.

4. Попов Г.М., Шафрановский И.И. Кристаллография. М.: Высшая школа, 1964. 370 с.

5. Шубников А.В. Симметрия и антисимметрия конечных фигур. М.: Изд-во АН СССР, 1951. 172 с.

6. Юшкин Н.П., Шафрановский И.И., Янулов К.П. Законы симметрии в минералогии. Л.: Наука, 1987. $336 \mathrm{c}$.

7. Heesch H. Über die vierdimensionalen Gruppen des dreidimensionalen Raumes // Z. Krist. 1930. Bd. 73. S. 325-345.

8. Voytekhovsky Y.L. On the real crystal octahedra // Acta Cryst. 2002. A 58. P. 622-623.

9. Voytekhovsky Y.L. How to name and order convex polyhedra // Acta Cryst. 2016. A72. Pt 5. P. 582-585.

10. Voytekhovsky Y.L., Stepenshchikov D.G. On the real crystal rhombododecahedra // Acta Cryst. 2004. A 60. P. 582-584. 